\title{
COVID-19 mortality risk factors in older people in a long-term care center
}

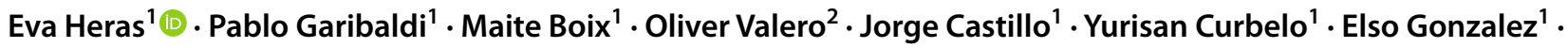 \\ Obilagilio Mendoza ${ }^{1} \cdot$ Maria Anglada $^{1} \cdot$ Joan Carles Miralles ${ }^{1} \cdot$ Petra Llull $^{1} \cdot$ Ricard Llovera $^{1} \cdot$ Josep M. Piqué ${ }^{1}$
}

Received: 18 August 2020 / Accepted: 11 November 2020 / Published online: 27 November 2020

(c) European Geriatric Medicine Society 2020

\section{Key summary points}

Aim COVID-19 mortality risk factors in older people from a long term care center.

Findings Male gender, low Barthel index, no pharmacological treatment and lymphocytopenia are independent mortality risk factors.

Message The independent prognostic factors identified in the present study can help to adjust the healthcare resources in this population in case of new outbreaks of the COVID-19 pandemic.

\begin{abstract}
Purpose Despite high rates of COVID-19 infection and increased related mortality have been reported among older adults admitted in long-term care facilities, a limited amount of information is available about the natural course of this pandemic and prognostic factors in such population. In the current study, we aimed to investigate the epidemiologic, demographics, clinical, or therapeutic factors that may predict the prognosis in a cohort of COVID-19 infected institutionalized older in a nursing home.

Methods We conducted a retrospective analysis of all COVID-19 confirmed institutionalized older in a nursing home from March 15 to June 5, 2020. Epidemiological, demographic, and frailty status before infection, and clinical, laboratory, treatment, and outcome data during infection were collected. We used bivariate analysis and multivariate logistic regression to identify risk factors for mortality.

Results The analysis comprised all 100 COVID-19 confirmed cases during the study period. The median age was 85 years; $62 \%$ were female. The case fatality rate was $20 \%$. In the bivariate analysis, male gender, fever, respiratory symptoms, severe cognitive decline, a low Barthel index, and lymphocytopenia were significantly associated with mortality. Patients treated with hydroxychloroquine plus azithromycin were related to a higher chance of survival than those without pharmacological treatment. Multivariate logistic regression analysis identified male gender, low Barthel index, no pharmacological treatment, and lymphocytopenia as independent risk factors associated with mortality.

Conclusions Male gender, low Barthel index, and lymphocytopenia are independent risk factors for COVID-19 mortality in institutionalized older patients in long-term care nursing homes. Treatment with hydroxychloroquine and azithromycin was associated with lower mortality in these patients.
\end{abstract}

\section{Introduction}

Eva Heras

eheras@saas.ad

1 Servei Andorra d'Atenció Sanitària, Carrer dels Escalls, Escaldes-Engordany, Les Escaldes, Andorra

2 Servei Estadistica Aplicada, Universitat Autònoma de Barcelona, Barcelona, Spain
While a great deal of information regarding the evolution of patients with COVID-19 infection [1-10] has been recorded in hospitals over the world since the onset of the novel coronavirus pandemic, only a few data have been published up to date regarding the course of the disease in patients admitted in long-term care facilities [11].

Studies by McMichael T.M. and Aron MM [11, 12] from a long-term care facility suggest that nursing home 
populations (e.g., older adults often with underlying chronic medical conditions) could be especially vulnerable to SARSCoV-2 infection and at higher risk for COVID-19-associated morbidity and mortality.

In the current study, we aimed to provide information about the clinical evolution of older patients infected by COVID-19 admitted in nursing homes and to identify prognostic mortality factors in such a population.

The study was approved by the Research Ethics Committee of the Andorran Healthcare Services.

\section{Methods}

\section{Study design}

The study was performed at a public nursing home from March 15 to June 5, 2020, identifying all the confirmed COVID-19 patients. This reference nursing home was transformed into a COVID-19 center for the admission of institutionalized seniors considered probable or confirmed cases of COVID-19 from the same center or other nursing homes. To this end, a joint action strategy was settled with the other nursing homes in the country, establishing a referral circuit to support them in the event of an outbreak of COVID-19 disease in their institution. Within $72 \mathrm{~h}$ of detecting the first COVID case, 35 residents were moved to a hotel to prevent further spread following medical criteria (residents with a low level of functional and cognitive dependence were transferred). In the different serological and PCR controls carried out, only three residents housed in the hotel had COVID-19 infection, and the mortality in this population was null.

\section{Data collection}

The following data were recorded for all patients: age, sex, date of admission, length of stay, the origin of the patient, dementia stage, Barthel index, Charlson comorbidity index, previous flu vaccination, clinical presentation, laboratory results, treatment, Reverse Transcription Polymerase Chain Reaction (RT-PCR) for SARS-CoV-2, hospital referral, mortality rate, and case fatality rate.

\section{Definitions}

Four categories of the patient's origin were defined: the reference nursing home, other nursing homes, home, or hotel. Patients from home belonged to a program of day care or home support for frail patients deployed by the same longterm care center.

Dementia was measured by the Global Deterioration Scale (GDS), developed by Dr. Reisberg [13], which consists of seven stages. 1: no cognitive decline, 2 : very mild cognitive decline, 3: mild cognitive decline, 4: moderate cognitive decline, 5: moderately severe cognitive decline, 6: moderately severe decline, 7: very severe cognitive decline.

Barthel index [14] is an ordinal scale of functional capacity used to measure performance in daily living activities, with values ranging from 0 (totally dependent) to 100 (totally independent). Proposed guidelines for interpreting Barthel index are: 0-15: totally dependent, 20-35: very dependent, 40-55: partially dependent, 60-75: minimally dependent, 80-100: totally independent [15].

Underlying diseases were considered as the presence of comorbid illness with the age adjusted Charlson comorbidity index [16], which predicts 10-year survival in patients with multiple comorbidities.

Patients were grouped into three clinical categories: asymptomatic, respiratory symptoms (rhinitis, pharyngitis, cough, expectoration, and dyspnea) and digestive symptoms (diarrhea). Fever was defined as an axillary temperature of at least $37.5^{\circ} \mathrm{C}$.

An analytical control was carried out, at least, on the admission and the discharge, recording the presence of lymphocytopenia, anemia, and or thrombocytopenia. Inflammation markers such as C-reactive protein (CRP), Ferritin, Dimer D, Lactate dehydrogenase (LDH), and troponin were also collected. Lymphocytopenia was defined as a total lymphocyte count of less than $1.0 \times 10^{9} / \mathrm{L}(1000 /$ $\mu \mathrm{L})$ and thrombocytopenia as a platelet count of less than $150 \times 10^{3}$ per $\mu \mathrm{L}$. The diagnosis of anemia in men was based on a hemoglobin of less than 13-14 g/dL; in women, less than $12-13 \mathrm{~g} / \mathrm{dL}$.

As for the treatment, five categories were defined: patients who received hydroxychloroquine and azithromycin, hydroxychloroquine only, hydroxychloroquine plus another antibiotic, beta-lactam, or quinolone antibiotics, and no treatment.

We also recorded the percentage of patients with two consecutive ( $48 \mathrm{~h}$ apart) negative molecular detection results for SARS-CoV-2 from a nasopharyngeal swab after 14 days of treatment with hydroxychloroquine and azithromycin.

Patients whose symptoms resolved and who had two consecutive (48 h apart) RT-PCR for SARS-CoV-2 negative were considered successfully treated and cured [17].

Four categories of discharge destination were defined: reference nursing home in non-COVID areas, other nursing homes, home, or death.

The mortality rate associated with COVID-19 in the center was defined as the death rate in the reference nursing home population.

The case fatality rate in the reference nursing center is the proportion of deaths from a COVID-19 disease compared to the total number of people diagnosed with the disease admitted in the center. 


\section{Statistical analysis}

Statistical analyses have been carried out to identify risk factors for mortality in patients with COVID 19. Bivariate tests have been performed between the qualitative and quantitative variables and the successfully treated variable COVID (yes/no). A chi-squared test was used for categorical variables and the $t$ test/Mann-Whitney test for quantitative variables. Additional bivariate analyses were performed to compare subject characteristics between treatment groups, to identify possible confounders. Variables with a $p$ value lower than 0.05 were included in a multivariate logistic regression model [18] to identify independent predictors of mortality. The final model was obtained after removing all non-statistically significant variables (backward selection procedure). Furthermore, a second multivariate regression model was adjusted, including only baseline variables to obtain a score to predict mortality independently of the effect of pharmacological treatment. ROC curves have been obtained to evaluate the fit of the models, and the area under the curve (AUC) has been calculated. The best cut-off point has been determined, and sensitivity and specified values have been obtained. Statistical analyses were performed with the SAS system version 9.4 (SAS Institute Inc., Cary, North Carolina, USA). The statistical significance level was set at 0.05 .

\section{Data availability}

The datasets generated and/or analysed for this study during the current study are available from the corresponding author upon request on reasonable request.

\section{Results}

The study population comprised 100 confirmed COVID-19 cases with a mean age of 85 (IQR 65-103) years. Sixtytwo percent were female, and the average length of stay was 22 days. Fifty-two patients were initially from the reference nursing home, 36 came from other nursing homes, nine from home, and three comings back from the hotel.

According to the global deterioration scale for the assessment of primary degenerative dementia, $73 \%$ of patients presented dementia, and $91.6 \%$ of dead had severe dementia (GDS6-GDS7). Seventy-six percent of patients had some level of functional dependence (Fig. 1). Clinically, 57\% had respiratory symptoms, and 39\% overcome the infection without any symptoms. Blood tests revealed anemia in 36 cases and lymphocytopenia in 38 patients.

Eighty-three percent received pharmacological treatment, mostly with hydroxychloroquine and azithromycin (70\%) (Fig. 2). Only five patients had diarrhea as a side effect related to hydroxychloroquine. Cardiac monitoring was

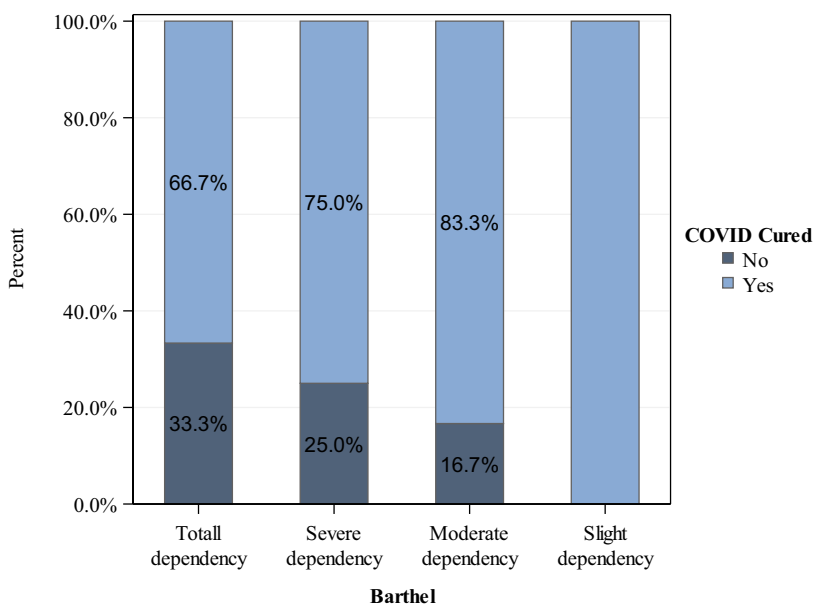

Fig. 1 Functional capacity and percentage of COVID-19 patients cured

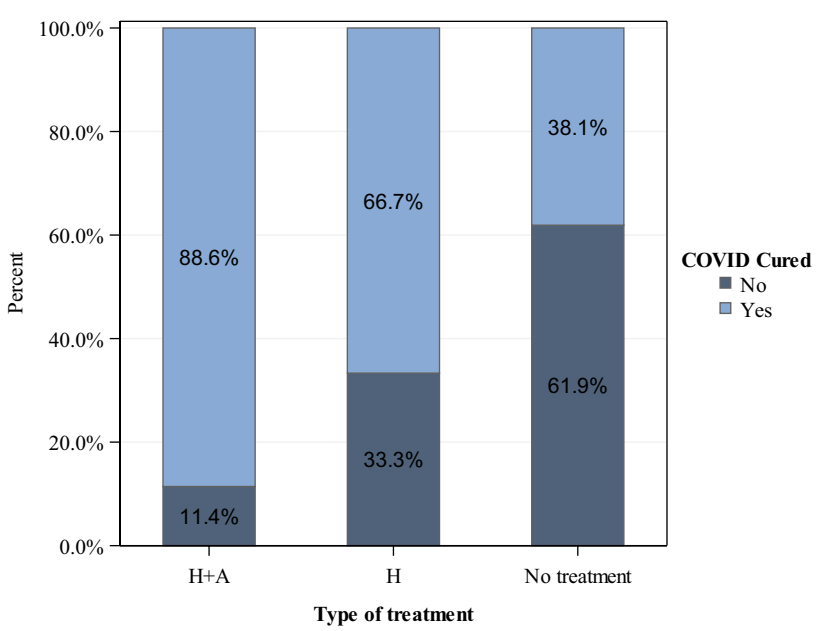

Fig. 2 Type of treatment and percentage of COVID-19 patients cured. $H+A$ hydroxychloroquine and azithromycin. $H$ hydroxychloroquine. No treatment includes Others: Beta-lactam or Quinolone antibiotics

performed by electrocardiogram, and no rhythm changes were observed with this treatment in any patient.

After 14 days, 12 patients had 2 negative molecular detection results for SARS-CoV-2 from a nasopharyngeal swab. Seventy-six patients were considered cured, and 24 died, of which 4 happened in the reference hospital. Thirty-five patients were discharged and sent back to non-COVID areas of Cedre's nursing home, 31 to other nursing homes, and 9 to home.

The mortality rate associated with COVID-19 only for the original residents of the reference nursing center was $14 \%$ (18 deaths/125 residents). The overall case fatality rate at the reference nursing home was 20\% (20 deaths/100 COVID-19 residents). 
In the bivariate analysis, the following factors showed a significantly greater risk of mortality among COVID-19 patients: male-gender, fever, respiratory symptoms, pharmacological treatment, type of treatment, serum therapy, oxygen therapy, dementia, Barthel index, lymphocytopenia, LDH, and D-dimer (Table 1).

No statistically significant differences were observed in Charlson's index, treatment started within $24 \mathrm{~h}$, anemia, low platelet count, ferritin, CRP, troponin levels, or previous flu vaccination.

Table 2 shows the results of the bivariate analysis results between treatment groups. Only 'treatment started within 24 h' was found statistically imbalanced between groups, but we did not find any relation between this variable and mortality. Temperature, serum therapy and D-dimer presented some differences between groups, although not statistically significant.

In the multivariate regression analysis, the independent risk factors associated with a higher COVID-19 related mortality were: male gender, type of treatment, Barthel index, and lymphocytopenia (Table 3). Although statistically significant differences were observed between LDH,
D-dimer and mortality values in the bivariate analysis, (as their levels increase, the risk of mortality increases) they were not included in the multivariate logistic regression due to the high number $(20 \%)$ of missings in these two parameters. A second multivariate analysis was performed including 'treatment started within $24 \mathrm{~h}$ ', temperature and serum therapy, and similar results as in the initial multivariate analysis were obtained.

To obtain a score that allows predicting COVID-19 progression from baseline, a new model was adjusted, excluding pharmacological treatment.

To calculate a prognostic score, the following formula was used:

Score $=1.4 \times($ Sex $=$ "Male" $)-0.04 \times$ Barthel +0.9 $\times($ Lymphopenia $=$ "Yes")

The higher the score, the higher was the probability of dying. We obtained an AUC of 0.85 (Fig. 3). The cut-off point that best ranks between patients who are successfully treated and those who are not cured is 0.2 , meaning all those patients with a score higher than 0.2 are most at risk of dying, with a specificity of $79.7 \%$ and a sensitivity of $80.0 \%$.
Table 1 Risk factors associated with COVID-19 mortality on bivariate analysis

\begin{tabular}{|c|c|c|c|}
\hline Variable & Cured & No cured & $p$ value \\
\hline Sex-male & $28.9(22)$ & $66.7(16)$ & $<0.001$ \\
\hline Age* & $86.5(7.6)$ & $86.1(8.9)$ & 0.888 \\
\hline Flu vaccine & $78.9(60)$ & $66.7(16)$ & 0.219 \\
\hline Temperature $>37.5^{\circ}$ & $52.6(40)$ & $79.2(19)$ & 0.021 \\
\hline Respiratory symptoms & $50.0(38)$ & $79.2(19)$ & 0.012 \\
\hline $\mathrm{H}^{\mathrm{a}}$ side effects & $2.8(2)$ & $13.0(3)$ & 0.090 \\
\hline Treatment $\mathrm{H}+\mathrm{A}^{\mathrm{a}}$ & $81.6(62)$ & $33.3(8)$ & $<0.001$ \\
\hline $\mathrm{H}^{\mathrm{b}}$ & $7.9(6)$ & $12.5(3)$ & \\
\hline No treatment ${ }^{\mathrm{c}}$ & $10.5(8)$ & $54.2(13)$ & \\
\hline Treatment started in the first $24 \mathrm{~h}$ & $39.5(30)$ & $37.5(9)$ & 0.863 \\
\hline Serum therapy & $43.4(33)$ & $79.2(19)$ & 0.002 \\
\hline Oxygen therapy & $50.0(38)$ & $95.8(23)$ & $<0.001$ \\
\hline Severe cognitive decline (GDS 5-7) & $67.1(51)$ & $91.7(22)$ & 0.018 \\
\hline Charlson* & $5.7(2.0)$ & $6.0(2.5)$ & 0.183 \\
\hline Barthel index* & $43.3(39.9)$ & $18.8(23.6)$ & 0.021 \\
\hline Severe/totally dependency (Barthel $\leq 60)$ & $65.8(50)$ & $91.7(22)$ & 0.014 \\
\hline Ferritin $(\log )^{*}$ & $6.0(1.1)$ & $6.6(1.3)$ & 0.106 \\
\hline LDH $(\log )^{*}$ & $6.2(0.3)$ & $6.4(0.4)$ & 0.033 \\
\hline Anemia & $43.2(32)$ & $26.7(4)$ & 0.233 \\
\hline Lymphocytopenia & $37.8(28)$ & $66.7(10)$ & 0.040 \\
\hline Thrombocytopenia & $25.7(19)$ & $33.3(5)$ & 0.542 \\
\hline D DIMER $(\log )^{*}$ & $0.31(0.86)$ & $1.04(0.97)$ & 0.015 \\
\hline $\mathrm{CRP}(\log )^{*}$ & $3.2(1.6)$ & $4.1(1.4)$ & 0.063 \\
\hline Troponin $(\log )^{*}$ & $3.5(0.7)$ & $4.4(1.1)$ & 0.092 \\
\hline
\end{tabular}

$\%(N)$ for categorical variables and means (SD) for quantitative $(*)$

$H+A^{a}$ hydroxychloroquine and azithromycin, $H^{b}$ hydroxychloroquine

No treatment ${ }^{\mathrm{c}}$ includes Others: Beta-lactam or Quinolone antibiotics 
Table 2 Bivariate analysis with treatment

\begin{tabular}{lcccc}
\hline Variable & $\mathrm{H}+\mathrm{A}^{\mathrm{a}}$ & $\mathrm{H}^{\mathrm{b}}$ & No treatment & $p$ value \\
\hline Sex-male & $37.1(26)$ & $22.2(2)$ & $47.6(10)$ & 0.407 \\
Age* & $86.3(7.3)$ & $88.3(8.1)$ & $85.9(9.6)$ & 0.727 \\
Flu vaccine & $75.7(53)$ & $100(9)$ & $66.7(14)$ & 0.146 \\
Tempera- & $54.3(38)$ & $55.6(5)$ & $76.2(16)$ & 0.197 \\
$\quad$ ture > 37.5 & & & & \\
Respiratory symp- & $55.7(39)$ & $66.7(6)$ & $57.1(12)$ & 0.823 \\
$\quad$ toms & & & & \\
$\mathrm{H}^{\mathrm{b}}$ side effects & $6.1(4)$ & $12.5(1)$ & $0(0)$ & 0.227 \\
Treatment 24 h & $48.8(34)$ & $11.1(1)$ & $19.0(4)$ & 0.021 \\
Serum therapy & $45.7(32)$ & $77.8(7)$ & $61.9(13)$ & 0.105 \\
Oxygen therapy & $57.1(40)$ & $77.8(7)$ & $66.7(14)$ & 0.409 \\
Severe cognitive & $70.0(49)$ & $77.8(7)$ & $81.0(17)$ & 0.578 \\
$\quad$ decline (GDS & & & & \\
5-7) & & & & \\
Charlson* & $5.7(2.1)$ & $5.7(2.5)$ & $5.9(2.1)$ & 0.876 \\
Barthel index* & $41.4(40.8)$ & $20.0(18.7)$ & $31.7(32.6)$ & 0.407 \\
Ferritin (log)* & $6.0(1.1)$ & $6.3(1.1)$ & $5.8(1.4)$ & 0.961 \\
LDH (log)* & $6.2(0.4)$ & $6.4(0.5)$ & $6.2(0.2)$ & 0.508 \\
Anemia & $40.3(27)$ & $50.0(4)$ & $35.7(5)$ & 0.806 \\
Lymphocytopenia & $41.8(28)$ & $37.5(3)$ & $50.0(7)$ & 0.813 \\
Thrombocytopenia & $20.9(14)$ & $50.0(4)$ & $42.9(6)$ & 0.088 \\
D DIMER (log)* & $0.30(0.9)$ & $1.01(1.1)$ & $0.70(1.0)$ & 0.102 \\
CRP (log)* & $3.4(1.6)$ & $3.5(1.8)$ & $3.6(1.8)$ & 0.895 \\
Troponin (log)* & $3.6(1.8)$ & $3.9(1.8)$ & $3.5(1.6)$ & 0.822 \\
\hline
\end{tabular}

$\%(N)$ for categorical variables and means (SD) for quantitative $(*)$

$H+A^{a}$ hydroxychloroquine and azithromycin, $H^{b}$ hydroxychloroquine

No treatment ${ }^{\mathrm{c}}$ includes Others: Beta-lactam or Quinolone antibiotics

Table 3 Risk factors associated with COVID-19 mortality on multivariate analysis

\begin{tabular}{lccc}
\hline Variable & B (SE) & OR & $p$ value \\
\hline Sex-male & $3.37(1.01)$ & 38.1 & 0.001 \\
Treatment H+ $\mathrm{A}^{\mathrm{a}}$ & $-3.12(1.04)$ & 0.044 & 0.004 \\
Treatment $\mathrm{H}^{\mathrm{b}}$ & $-1.13(1.25)$ & 0.32 & 0.369 \\
Barthel's index & $-0.047(0.017)$ & 0.92 & 0.006 \\
Lymphopenia & $1.88(0.92)$ & 6.55 & 0.039 \\
\hline
\end{tabular}

$H+A^{a}$ hydroxychloroquine and azithromycin, $H^{b}$ hydroxychloroquine Treatment considers 'No treatment' as reference

\section{Discussion}

With the outbreak of COVID-19, the Andorran Healthcare Service acted quickly and proactively to control the pandemic in nursing homes. The early reaction transforming the reference nursing home into an intermediate healthcare facility and moving to a hotel 35 healthy residents from this center with a low level of functional and cognitive

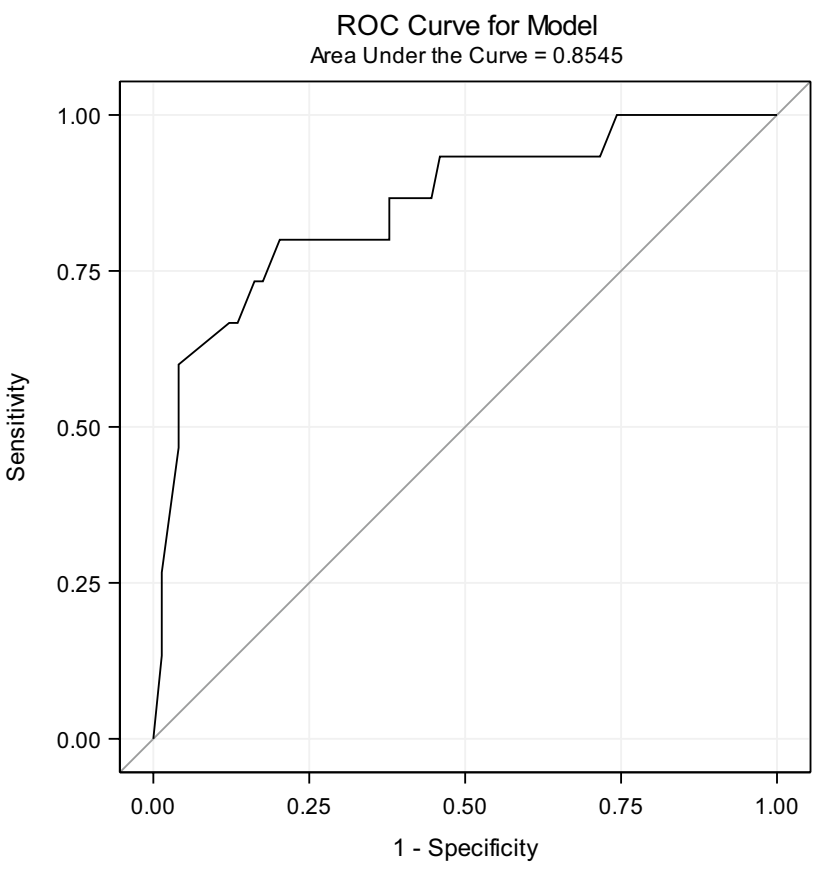

Fig. 3 Prognostic COVID-19 mortality score-ROC curve for model

dependence, probably avoided excess mortality since none of the people transferred to the hotel died. One hundred COVID-19 older patients were admitted to the reference intermediate care nursing home, and they received the same care as they would get in hospital wards, $76 \%$ of cases were cured. The case fatality rate in those remaining in the nursing home was lower (20\%) than that reported in other settings $(34 \%)[11,12]$ despite being mostly patients with high functional dependence. Seven patients were transferred to a tertiary care hospital for presenting medical criteria for mechanical respiratory ventilation.

As far as we know, this is the first reported study describing how a nursing home was transformed into an intermediate care facility to face the COVID-19 outbreak and analyzing which prognostic factors could predict infection-related mortality in this segment of the older population, despite the deployment of a significant amount of medical resources.

Several factors related to gender, respiratory symptoms, supportive and specific therapy, cognitive and functional deterioration, and inflammatory and immune factors were associated with mortality in the bivariate analysis. In the multivariate logistic regression analysis, only male gender, Barthel index, lymphocytopenia, and hydroxychloroquine plus azithromycin were identified as independent factors for mortality. Variables that presented more differences between treatment groups were included in a second multivariate model to evaluate potential confounding factors, and similar results were obtained. 
Many reports have outlined that, although rates of infection are similar among men and women, men are at higher risk of death from the COVID-19 infection [3, 19]. The explanation for that phenomenon is unclear, although previous studies have suggested that women are less susceptible to viral infection, possibly because of the protection of $\mathrm{X}$ chromosome and sex hormones, which play an essential role in innate and adaptive immunity [20].

The institutionalized older persons present a cognitive and functional decline that may worsen with acute illness such as COVID-19 [21]. This study reveals a direct relationship between decreased functional capacity measured by the Barthel index and mortality. Functional status in older people has been identified as a prognostic mortality factor in respiratory infections [22]. The current study is the first to prove a direct relationship between the Barthel index and mortality in COVID-19 infected patients.

Contrary to what was expected, we did not find any relationship between clinical comorbidity and mortality in these patients, a factor that is usually present in studies evaluating mortality for other causes in older people [23-25], and it was also relevant in previous reports of COVID-19 infection in China [5, 6, 26]. Moreover, flu vaccination did not have any impact on mortality either.

The other independent risk factor detected was lymphocytopenia, a common finding in COVID-19 infected patients that has been previously related to mortality $[4,27,28]$. This could be due to a direct effect of the virus or the consequence of the cytokine-mediated inflammatory cascade inducing lymphocytes migration [27]. In any case, T-lymphocytes deficiency or dysregulation may reflect oversized immune reactions that can contribute to disease severity and mortality [29].

Regarding the treatment, hydroxychloroquine and azithromycin were prescribed for 5 days as the initial treatment for all patients. Before and during this initial treatment period, electrocardiograms were performed to monitor patient's tolerance to the treatment. When this pharmacological combination was contraindicated based on electrocardiographic alterations, either hydroxychloroquine alone or beta-lactams were prescribed instead.

It is worth highlighting that the multivariate logistic regression analysis identified hydroxychloroquine plus azithromycin treatment as an independent factor favoring survival compared with no treatment or other treatments. At present, contradictory results have been published regarding the effectiveness of this treatment in COVID-19-infected patients and the associated risk of adverse events using those medications [30-34]. It might be possible that this treatment could only work when used early and in a particular group of patients with similar specific characteristics as in the present study. In any case, only randomized clinical trials could definitively clear these uncertainties.
Using only those independent risk factors identified in the multivariate analysis, which could be easily collected at onset of the disease infection, we have elaborated a score that, beyond the potential beneficial effect of pharmacological treatment, may be able to predict mortality with reasonable specificity and sensitivity.

This study has some limitations. First, it is a retrospective study, and some missing laboratory data precluded to include LDH and D-dimer parameters in the multivariate regression analysis. Second, the advanced age of all patients included has probably been prevented from identifying this parameter as a prognostic factor for mortality. Third, the high prevalence of dementia and functional dependence among the patients included may have generated results that might not be precisely reproducible in other cohorts of older patients with better functional capabilities. Finally, it would be interesting to evaluate the presence of interactions between variables for which a bigger sample size would be required.

\section{Conclusion}

In summary, this study describes the feasibility of transforming a nursing home into an intermediate healthcare facility to face an epidemic outbreak of COVID-19 in this setting and limiting referrals to a tertiary care hospital. To take care of COVID-19-infected older person, the independent prognostic factors identified in the present study can be of enormous help to adjust the adequate healthcare resource provision and to limitate therapeutic efforts in case of new outbreaks of the pandemic.

Acknowledgements We respectfully and sincerely thank all front-line healthcare workers from the reference nursing home for their hard work and sacrifice in the face of this outbreak.

Author contribution EH contributed to the study design, data collection, interpretation of the data, writing and revising the manuscript and accepts responsibility for the corresponding author. PG, MB, JC, YC, EG, OM, MA, JCM, PL, RL contributed to the data collection, data interpretation, and revising of the manuscript. OV carried out the data analysis, data interpretation, and editing of the manuscript. JMP contributed to the interpretation of the data, writing, and revising of the manuscript. All authors read and approved the final manuscript.

\section{Compliance with ethical standards}

Conflict of interest We declare no competing interests.

Ethical approval This study was approved by the Research Ethics Committe of the Andorran Healthcare System. 


\section{References}

1. Zhou F, Yu T, Du R, Fan G, Liu Y, Xiang J et al (2020) Clinical course and risk factors for mortality of adult in-patients with COVID-19 in Wuhan, China: a retrospective study. Lancet 395:1054-1062

2. Richardson S, Hirsch JS, Narasimhan M, Crawford JM, McGinn T, Davidson KW et al (2020) Presenting characteristics, comorbidities, and outcomes among 5700 patients hospitalized with COVID-19 in the New York City area. JAMA 323:2052-2059

3. Xiaochen L, Shuyun XU, Muqing Y, Wang K, Tao Y, Zhou Y et al (2020) Risk-factors for severity and mortality in adult COVID-19 inpatients in Wuhan. J Allergy Clin Immunol 146(1):110-118

4. Chen T, Wu D, Chen H, Yan W, Yang D, Chen G et al (2020) Clinical characteristics of 113 deceassed patients with coronavirus disease 2019: retrospective study. BMJ 368:m1091

5. Tian W, Jiang W, Yao J, Nicholson CJ, Li RH, Haakon H et al (2020) Predictors of mortality in hospitalized COVID-19 patients: a systematic review and meta-analysis. J Med Virol. https://doi. org/10.1002/jmv.26050

6. Du RH, Liang LR, Yang CQ, Wang W, Cao TZ, Li M et al (2020) Predictors of mortality for patients with COVID-19 pneumonia caused by SARS-CoV-2: a prospective cohort study. Eur Respir J 55(5):2000524. https://doi.org/10.1183/13993003.00524-2020

7. Na Z, Ding Z, Wen W (2020) Clinical features of patients infected with 2019 novel coronavirus in Wuhan, China. Lancet 395:497-605

8. Huang C, Wang Y, Li X, Ren L, Zhao J, Hu Y (2020) Clinical features of patients infected with 2019 novel coronavirus in Wuhan, China. Lancet 395:497-506

9. Zhou F, Yu T, Du R, Fan G, Liu Y, Liu Z (2020) Clinical course and risk factors for mortality of adult inpatients with COVID19 in Wuhan, China: a retrospective cohort study. Lancet 395:1054-1062

10. Chen N, Zhou M, Dong X et al (2020) Epidemiological and clinical characteristics of 99 cases of 2019 novel coronavirus pneumonia in Wuhan, China: a descriptive study. Lancet 395:507-513

11. McMichael TM, Currie DW, Clark S, Pogosjans S, Kay M, Schwartz NG et al (2020) Epidemiology of COVID-19 in a longterm care facility in King County, Washington. N Engl J Med 382:2005-2011

12. Arons MM, Hatfield KM, Redy SC, Kimball A, James A, Jacobs JR et al (2020) Presymptomatic SARS-Cov-2 infections and transmission in a skilled nursing facility. N Eng J Med 382:2081-2089

13. Reisberg B, Ferris SH, de Leon MJ, Crook T (1982) The global deterioration scale for assessment of primary degenerative dementia. Am J Psychiatry 139:1136-1139

14. Mahoney FI, Barthel DW (1965) Functional evaluation: the Barthel Index. Md State Med J 14:61-65

15. Shah S, Vanclay F, Cooper B (1989) Improving the sensitivity of the Barthel Index for stroke rehabilitation. J Clin Epidemiol 42:703-709

16. Charlson ME, Pompei P, Ales KL et al (1987) A new method of classifying prognostic comorbidity in longitudinal studies: development and validation. J Chronic Dis 40(5):373-383

17. European Centre for Disease Prevention and Control (2020) Novel Coronavirus (SARS-CoV-2). Discharge criteria for confirmed COVID-19 cases. https://www.ecdc.europa.eu/sites/default/files /documents/COVID-19-Discharge-criteria.pdf

18. Hosmer DW, Lemeshow S (2013) Applied logistic regression 3rd edition. John Wiley \& Sons, Inc.
19. Jin JM, Bai P, He W, Wu F, Liu XF, Han DM et al (2020) Gender differences in patients with COVID-19: focus on severity and mortality. Front Public Health 8:152. https://doi.org/10.3389/ fpubh.2020.00152

20. Gal-Oz ST, Maier B, Yoshida H (2019) ImmGen report: sexual dimorphism in the immune system transcriptome. Nat Commun 10(1):4295. https://doi.org/10.1038/s41467-019-12348-6

21. Wang H, Li T, Barbarino P, Gauthier S (2020) Dementia care during COVID-19. Lancet 395(10231):1190-1191

22. Corrao S, Argano C, Natoli G, Nobili A, Corazza GR, Mannucci PM et al (2018) Disability and not diabetes is a strong predictor of mortality in oldest patients hospitalized with pneumonia. Eur J Intern Med 54:53-59

23. Quan H, Li B, Couris CM, Fushimi K, Graham P, Hider P et al (2011) Updating and validating the Charlson comorbidity index and score for risk adjustment in hospital discharge abstracts using data from 6 countries. Am J Epidemiol 173:676-682

24. Onder G, Rezza G, Brusaferro S (2020) Case-fatality rate and characteristics of patients dying in relation to COVID-19 in Italy. JAMA 323(18):1775-1776

25. Ruan Q, Yang K, Wang W, Jiang L, Song J (2020) Clinical predictors of mortality due to COVID19 based on an analysis of data of 150 patients from Wuhan, China. Intensive Care Med 46(5):846-848

26. Wang L, He W, Yu X, Hu D, Bao M, Liu H et al (2020) Coronavirus disease 2019 in elderly patients: charateristics and prognostic factors based on 4-week follow-up. J Infect 80:639-645

27. Qin C, Zhou L, Hu Z, Zhang S, Yang S, Tao Y et al (2020) Dysregulation of immune response in patients with COVID-19 in Wuhan, China. Clin Infect Dis 71(15):762-768

28. Lippi G, Plebani M, Henry BM (2020) Thrombocytopenia is associated with severe coronavirus disease 2019(COVID-19) infections: a meta-analysis. Clin Chim Acta 506:145-148

29. Pedersen SF, Ho Ch (2020) SARS-Cov-2: a storm is raging. J Clin Invest 130:2202-2205

30. Chowdhury MS, Rathod J, Gernsheimer J (2020) A rapid systematic review of clinical trials utilizing chloroquine and hydroxychloroquine as a treatment for COVID-19. Acad Emerg Med 27:493-504

31. Gautret P, Lagier JC, Parola P, Hoang VT, Meddeb L, Mailhe M et al (2020) Hydroxychloroquine and azithromycin as a treatment of COVID-19: results of an open-label non-randomized clinical trial. Int J Antimicrob Agents 56(1):105949

32. Rosenberg ES, Dufort EM, Udo T, Wilberschied LA, Kumar J, Tesoriero J et al (2020) Association of treatment with hydroxychloroquines or azithromycin with in-hospital mortality in patients with COVID-19 in New York State. JAMA. https://doi. org/10.1001/jama.2020.8630

33. Cortegiani A, Ingoglia $\mathrm{G}$, Ippolito $\mathrm{M}$, Giarratano A, Einav $\mathrm{S}$ (2020) A systematic review on the efficacy and safety of chloroquine for the treatment of COVID-19. J Crit Care 57:279-283

34. Gao J, Tian Z, Yang X (2020) Breakthrough: chloroquine phosphate has shown apparent efficacy in treatment of COVID-19 associated pneumonia in clinical studies. Biosci Trends 14(1):7273. https://doi.org/10.5582/bst.2020.01047

Publisher's Note Springer Nature remains neutral with regard to jurisdictional claims in published maps and institutional affiliations. 